\title{
Substitution de la farine de poisson par la farine d'asticots séchés dans le régime du rat en croissance : risques pathologiques?
}

\author{
K.G.M. BOUAFOU ${ }^{1 *}$, B.A. KONAN ${ }^{2}$, A. MEITE ${ }^{2}$, K.G. KOUAME ${ }^{2}$ and \\ S. KATI-COULIBALLY ${ }^{2}$ \\ ${ }^{I}$ Section des Sciences de la Vie et de la Terre, Département des Sciences et Technologie, Ecole Normale \\ Supérieure d'Abidjan (Côte d'Ivoire), 22 B.P. 582 Abidjan 22. \\ ${ }^{2}$ Laboratoire de Nutrition et Pharmacologie, UFR-Biosciences, Université de Cocody (Côte d'Ivoire), \\ 22 B.P. 582 Abidjan 22. \\ *Auteur correspondant, E- mail : bouafou_k@yahoo.fr, Tel (225) 665292 38/(225) 40309965
}

\section{RESUME}

Deux groupes de dix rats en croissance sont nourris durant 15 jours, avec deux régimes alimentaires ne différant que par la qualité de leurs protéines animales. L'un contient $10 \%$ de farine de poisson (témoin) et l'autre, $10 \%$ de farine d'asticots séchés (FAS). Le dosage des paramètres biochimiques plasmatiques et la biométrie des reins et des foies sont effectués chez tous les rats en fin d'expérience. Aucune pathologie ni anomalie physiologique n'a été décelée à travers les paramètres biochimiques plasmatiques. Mais, la biométrie des organes révèle chez les rats sous régime FAS, une diminution de $6,60 \%$ du poids des reins et une augmentation de $10,60 \%$ du poids des foies, en comparaison aux sujets témoins. Ces résultats pourraient présager une pathologie ou une perturbation du métabolisme nutritionnel de ces organes.

(C) 2011 International Formulae Group. All rights reserved.

Mots clés : Protéines animales, alimentation, paramètres biochimiques plasmatiques, biométrie des organes.

\section{INTRODUCTION}

La farine d'asticots séchés (FAS) s'est révélée, au plan strictement nutritionnel, une source appréciable de protéines alimentaires chez les rats en croissance (Bouafou et al., 2007, 2008). Cependant, pour prescrire sans risques son emploi dans l'alimentation animale, il est nécessaire de mener des investigations complémentaires, car la FAS est une source non conventionnelle de protéines alimentaires. Cette étude vise à déceler d'éventuelles pathologies dans les foies et les reins des rats en croissance, nourris à la FAS, par le dosage de leurs paramètres biochimiques plasmatiques et par la biométrie de leurs organes.

En effet, l'intérêt du dosage des paramètres biochimiques plasmatiques dans les études de nutrition est bien établi (Séronie et al., 2004). L'excès ou le déficit de production d'une substance synthétisée par un organe est révélateur d'un dysfonctionnement de celui-ci. C'est une voie d'exploration indirecte des organes dits régulateurs du 
métabolisme et un moyen d'appréciation du métabolisme des nutriments. De même, dans les études de nutrition, l'évolution pondérale de certains organes impliqués dans la digestion et l'absorption des nutriments, constitue une voie indirecte de leur exploration (Adrian et al., 1991). L'atrophie ou l'hypertrophie d'un organe, si elle n'est pas physiologique, peut être l'indice d'une pathologie de celui-ci ou d'une perturbation du métabolisme nutritionnel en son sein.

\section{MATERIEL ET METHODES}

Certains matériel et méthodes de cette étude sont les mêmes que ceux utilisés par Bouafou et al. (2007, 2008).

\section{Animaux et logements}

Des rats mâles de race Wistar en croissance (poids moyen : $61 \mathrm{~g}$ ) ont été logés dans des cages à métabolisme individuelles. Ces cages ont été munies de râteliers et de biberons pour alimenter et abreuver les animaux.

Production des asticots, de la farine d'asticots séchés (FAS), de la farine de poisson (témoin) et de la farine de maïs

Des épluchures d'ignames et des restes de poissons frais disposés en couches dans une demi-barrique, ont été exposés aux mouches. Après 24 heures d'ensemencement des substrats, la demi-barrique a été couverte. Quatre jours plus tard, les asticots ont été récoltés dans de l'eau bouillante. Les asticots récoltés ont été séchés à l'étuve à $70{ }^{\circ} \mathrm{C}$ pendant 24 heures. Ils ont été ensuite moulus dans un mixer Moulinex pour obtenir la farine d'asticots séchés.

La farine de poisson a été produite de la même manière que la FAS, à partir de poissons (hareng) frais, achetés dans une poissonnerie.

La farine de maïs utilisée dans cette expérience est celle du commerce. Elle est blanche. Le maïs est couramment utilisé pour l'alimentation animale. C'est le principal pourvoyeur d'énergie du régime, du fait de ses teneurs élevées en amidon et en acides gras (Bouafou, 2007).

\section{Formulation des régimes alimentaires}

Les compositions chimiques de la FAS, de la farine de poisson et de la farine de maïs ont été consignées dans le Tableau 1. Les régimes alimentaires ont été préparés selon la méthode de Pawlak et Pion (1968) par Bouafou et al. (2007, 2008). Deux régimes isocaloriques $(4200 \mathrm{kcal} / \mathrm{kg}$ de matière sèche) et isoprotéiques $(16 \%$, soit $160 \mathrm{~g} / \mathrm{kg}$ de matière sèche) ont été préparés (Tableau 2).

Le régime témoin contenait $10 \%$ de protéines animales, constituées de farine de poisson. Le régime FAS (ou test) a été obtenu en remplaçant dans le régime témoin, la farine de poisson par la FAS.

\section{Conditions expérimentales et constitution des lots de rats}

La salle d'expérience avait une température de $26{ }^{\circ} \mathrm{C}$, avec une hygrométrie comprise entre 70 et $80 \%$.

Deux lots de dix rats en croissance ont été soumis chacun aux régimes alimentaires confectionnés.

\section{Conduite de l'expérience}

L'expérimentation animale s'est faite selon la méthode d'Adrian et al. (1991), reprise par Bouafou et al. (2007, 2008). Les régimes sont distribués ad libitum une fois par jour (de 6 h 30 min à 7 h 30 min) sous forme de purée. L'eau est servie à volonté et renouvelée tous les trois jours.

\section{Dosage des paramètres biochimiques plasmatiques \\ Ces paramètres ont été dosés sur du} sérum obtenu après centrifugation du sang entier, au Laboratoire de Biochimie Médicale de l'Université de Cocody-Abidjan. Les dosages se sont conformés aux prescriptions des réactifs des kits du groupe espagnol 
SPINREACT S.A. (Fawcett et Scoot, 1960; Budesinky, 1969; Dingeon et al., 1975; Young et al., 1975). La valeur de chacun des paramètres biochimiques plasmatiques (en $\mathrm{mg} / \mathrm{dl}$ ) a été déterminée à partir de la relation suivante :

$\mathrm{P}=[$ D.O. écht/D.O. standard $] \mathrm{x}$ concentration standard, où $\mathrm{P}$ est le paramètre biochimique plasmatique à doser, la D.O. écht (densité optique de l'échantillon) est égale à $610 \mathrm{~nm}$ et la concentration standard est de $200 \mathrm{mg} / \mathrm{dl}$.

\section{Prélèvement et pesée d'organes}

A la fin de la période expérimentale, les rats ont été anesthésiés, puis sacrifiés pour le prélèvement des reins et du foie (Bouafou, 2007).

Les organes prélevés ont été déshumidifiés sur du papier hygiénique, puis pesés sur une balance de précision Sartorius de sensibilité $0,001 \mathrm{~g}$.

\section{Analyse statistique}

Les comparaisons des valeurs des paramètres biochimiques plasmatiques ont été effectuées à partir du logiciel STATISTICA, version 6.0, par le test de Newman Keuls à 0,01 .

\section{RESULTATS}

\section{Etude des paramètres biochimiques plasmatiques \\ Urée et créatinine}

L'urémie et la créatinémie de tous les animaux ont peu varié. Les valeurs observées étaient statistiquement égales $(\mathrm{p}>0,01)$. L'urémie a été de $0,27 \pm 0,07 \mathrm{mg} / \mathrm{ml}$ pour les jeunes rats témoins et de $0,20 \pm 0,05 \mathrm{mg} / \mathrm{ml}$ pour ceux sous régime FAS.

La créatinémie des jeunes rats témoins a été de $0,69 \pm 0,06 \mathrm{mg} / \mathrm{ml}$. Celle des jeunes rats testés a été de $0,84 \pm 0,13 \mathrm{mg} / \mathrm{ml}$.

\section{Triglycérides et cholestérol}

Le taux sanguin de triglycérides des rats témoins a été de $0,61 \pm 0,18 \mathrm{mg} / \mathrm{ml}$. Celui des jeunes animaux ingérant la FAS a été de $0,44 \pm 0,12 \mathrm{mg} / \mathrm{ml}$. Il n'y avait pas de différence significative $(p>0,01)$ entre les triglycéridémies de tous les jeunes rats.

La cholestérolémie des sujets témoins a été de 4,00 $\pm 0,00 \mathrm{mg} / \mathrm{ml}$, tandis que celle des jeunes rats testés s'élevait à $3,16 \pm 0,77$ $\mathrm{mg} / \mathrm{ml}$. Cette dernière est significativement inférieure $(\mathrm{p}<0,01)$ à celle des jeunes rats témoins.

\section{Glucose}

Le dosage de la glycémie des jeunes rats témoins a donné $2,13 \pm 0,31 \mathrm{mg} / \mathrm{ml}$ et celui des sujets testés, $2,54 \pm 0,30 \mathrm{mg} / \mathrm{ml}$. Les glycémies des jeunes rats témoins et testés n'ont présenté aucune différence statistiquement significative.

\section{Rapport calcium/phosphore}

Le rapport calcium/phosphore des jeunes rats témoins a été de $0,88 \pm 0,12$. Celui des rats testés a été de $0,82 \pm 0,12$. Ils ne présentaient pas de différence significative ( $>0,01)$.

\section{Evolution pondérale des reins et du foie Reins}

Le poids moyen des reins, rapporté au poids corporel des rats témoins a été de $0,76 \pm$ 0,06 . Celui de leurs congénères traités lui est statistiquement $(p>0,01)$ inférieur. Ainsi, le poids moyen des reins rapportés au poids corporel des rats sous régime FAS est de 0,75 $\pm 0,03$. Leurs reins ont subi une diminution pondérale de l'ordre de $6,60 \%$.

Foie

Le poids moyen du foie rapporté au poids corporel des rats soumis au régime FAS est apparu statistiquement supérieur $(\mathrm{p}<0,01)$ à celui des animaux témoins, soit $4,36 \pm 0,19$ contre 3,94 $\pm 0,19$. Le taux d'augmentation du poids moyen du foie des rats du régime FAS est de $10,60 \%$. 
Tableau 1: Compositions chimiques (\% de matière sèche) de la farine d'asticots séchés, de la farine de poisson et de la farine de maïs.

\begin{tabular}{llll}
\hline Composantes & Farine d'asticots séchés & $\begin{array}{l}\text { Farine de poisson } \\
\text { (hareng) du } \\
\text { commerce }\end{array}$ & $\begin{array}{l}\text { Farine de maïs blanc } \\
\text { du commerce }\end{array}$ \\
\hline Matière sèche & $92,51 \pm 5,23$ & $89,70 \pm 6,33$ & $88,00 \pm 7,40$ \\
$\begin{array}{l}\text { Protéine } \\
\text { Matière grasse }\end{array}$ & $52,23 \pm 2,80$ & $61,29 \pm 3,14$ & $9,46 \pm 2,88$ \\
& $24,43 \pm 3,11$ & $9,3 \pm 0,81$ & $2,52 \pm 0,06$ \\
Extractifs & & & \\
azotés & $16,01 \pm 0,92$ & $18,21 \pm 2,01$ & $69,00 \pm 1,55$ \\
$\begin{array}{l}\text { Cendre } \\
\text { Sodium }\end{array}$ & $7,33 \pm 0,64$ & $11,00 \pm 0,90$ & $1,45 \pm 0,08$ \\
$\begin{array}{l}\text { Potassium } \\
\text { Calcium }\end{array}$ & $0,51 \pm 0,07$ & $1,00 \pm 0,05$ & $0,03 \pm 0,01$ \\
\hline Son & $0,10 \pm 0,03$ & $0,70 \pm 0,06$ & $0,05 \pm 0,01$ \\
\hline
\end{tabular}

Source: Bouafou (2007)

Tableau 2: Formulation des régimes expérimentaux.

\begin{tabular}{|c|c|c|c|c|}
\hline \multirow{2}{*}{\multicolumn{2}{|c|}{ Intrants alimentaires }} & \multirow{2}{*}{$\begin{array}{c}\text { \% de } \\
\text { matière sèche } \\
\text { (MS) }\end{array}$} & \multicolumn{2}{|c|}{ Régimes alimentaires } \\
\hline & & & $\begin{array}{l}\text { Farine de } \\
\text { poisson } \\
\text { (témoin) }\end{array}$ & $\begin{array}{c}\text { Farine } \\
\text { d'asticots } \\
\text { séchés (FAS) }\end{array}$ \\
\hline \multirow[t]{3}{*}{ Protéine } & $\begin{array}{l}\text { Farine de } \\
\text { maïs }\end{array}$ & $6 \%$ & 633,91 & 633,91 \\
\hline & Farine & $10 \%$ & 163,15 & 0 \\
\hline & & & 0 & 191,46 \\
\hline \multicolumn{2}{|c|}{$\begin{array}{l}\text { Complément minéral } \\
\text { complet UAR-205 }\end{array}$} & $7 \%$ & 70 & 70 \\
\hline \multicolumn{2}{|c|}{ Complément vitaminé } & $1 \%$ & & \\
\hline \multicolumn{2}{|c|}{ UAR-200 } & & 10 & 10 \\
\hline \multicolumn{2}{|c|}{ Cellulose et agar-agar } & $3 \%$ & 9,47 & 9,47 \\
\hline \multicolumn{2}{|c|}{ Huile de maïs } & & 116 & 116 \\
\hline \multicolumn{2}{|c|}{ Total MS en $\mathrm{g}$} & & 1000 & 1000 \\
\hline \multicolumn{2}{|c|}{$\begin{array}{l}\text { Energie brute en } \mathrm{kcal} / \mathrm{kg} \\
\text { de MS }\end{array}$} & & 4200 & 4200 \\
\hline
\end{tabular}

N.B. : L'énergie brute des régimes alimentaires a été calculée en se référant aux valeurs de combustion des différents nutriments sur la base de $4 \mathrm{kcal}$ pour $1 \mathrm{~g}$ de protéine, $4 \mathrm{kcal}$ pour $1 \mathrm{~g}$ de glucide et $9 \mathrm{kcal}$ pour $1 \mathrm{~g}$ de lipide.

\section{DISCUSSION}

\section{Paramètres biochimiques plasmatiques}

Globalement, les valeurs des paramètres biochimiques plasmatiques des jeunes rats traités et témoins n'ont pas présenté de différence significative $(\mathrm{p}>0,01)$.

L'urémie normale $(0,27 \pm 0,07 \mathrm{mg} / \mathrm{ml})$ notée chez les animaux témoins est statistiquement semblable à celle des jeunes rats traités $(0,20 \pm 0,05 \mathrm{mg} / \mathrm{ml})$. Ce résultat atteste à priori, d'une bonne physiologie rénale (Abuelo, 1990 ; Mogensen et al., 1995). La créatinémie est utilisée pour l'évaluation de routine de la fonction rénale (Séronie et al., 2004). La créatinémie normale des jeunes rats ingérant la FAS n'a pas révélé 
un dysfonctionnement rénal (Balasubramaiam, 2003).

Le rapport normal $\mathrm{Ca} / \mathrm{P}$ plasmatique est l'indice d'un bon métabolisme de ces deux minéraux par les reins. Une modification de leurs taux dans le sang peut laisser soupçonner la formation de calculs rénaux (Williams, 1994; Brunette, 1997).

Les taux plasmatiques de cholestérol des jeunes rats témoins et traités ont présenté une différence statistiquement significative. Cette information peut conduire à suspecter des troubles hépatiques plus ou moins importants. En effet, la cholestérolémie et la triglycéridémie, régulées par le foie, sont modifiées en cas de maladies hépatiques (Anonyme, 1985).

En outre, il est remarqué que les jeunes rats ayant ingéré la FAS ont eu une glycémie $(2,54 \pm 0,30 \mathrm{mg} / \mathrm{ml})$ élevée, en comparaison à celle des jeunes sujets témoins $(2,13 \pm 0,31$ $\mathrm{mg} / \mathrm{ml}$ ), quoique statistiquement égales. Cela peut être révélateur d'une pathologie hépatique ou rénale dont l'ampleur est à découvrir. Il a été clairement prouvé qu'une glycémie élevée peut être la cause d'un déficit de sécrétion d'insuline, d'un mauvais stockage du glucose par le foie ou d'une atteinte rénale. Malgré tout ce qui précède, le dosage de tous ces paramètres n'autorise pas à soupçonner un dysfonctionnement des reins et des foies des rats sous aliments FAS.

\section{Biométrie des organes}

$\mathrm{Au}$ contraire des paramètres biochimiques plasmatiques, la biométrie des organes a décelé la diminution de poids des reins et l'augmentation du poids des foies des rats sous régimes FAS. Il pourrait s'agir respectivement d'une hypoplasie rénale doublée d'une hyperplasie hépatique chez ces animaux.

$\mathrm{Au}$ niveau des foies, les modifications de poids $(10,60 \%$ d'augmentation) sont plus importantes qu'au niveau des reins $(6,60 \%$ de diminution). En effet, le foie est un lieu d'intenses activités métaboliques (catabolisme et synthèse) des protéines, des lipides et des glucides tandis que le rein ne joue qu'un rôle de régulation hydroélectrolytique essentiellement.

L'hypertrophie hépatique, observées chez les jeunes rats ingérant la FAS, peut laisser suggérer que ce régime renferme des substances difficilement métabolisables, imposant donc à ces organes un surcroît d'activité (Adrian et al., 1991). Quant à l'atrophie rénale subie par les rats sous régime FAS, elle peut traduire une manifestation de pathologies plus ou moins graves des organes concernés (Williams, 1994). Ces pathologies pourraient être la conséquence de l'ingestion de substances antinutritionnelles contenues éventuellement dans la FAS (Satish et al., 1984).

Ces modifications de poids d'organes ont été déjà observées dans d'autres travaux (Bouafou et al., 2008). Finke et al. (1989) ont montré que des rats nourris avec des régimes aux taux élevés de farine de criquets comme seule source de protéine, ont eu la diarrhée et leurs estomac et côlon se sont distendus. De plus, deux rats sur cinq sont morts dans le lot nourri au plus fort taux de protéine de criquet.

Par ailleurs, il apparaît que ces modifications de poids d'organes constatées chez les rats traités ne sont pas corrélées aux paramètres biochimiques plasmatiques, car ces derniers ont des valeurs normales.

En définitive, le dosage des paramètres biochimiques plasmatiques et la biométrie des reins et des foies n'ont pas permis de révéler avec certitude, des pathologies dans lesdits organes chez les rats ingérant la FAS à $10 \%$ dans leur régime.

\section{Conclusion}

L'exploration par le dosage des paramètres biochimiques plasmatiques, des reins et des foies des rats ayant consommé un régime incorporant $10 \%$ de FAS, n'a pas décelé globalement, des anomalies ou des pathologies de ces organes. Les paramètres biochimiques plasmatiques de ces rats sont normaux en comparaison de ceux de leurs congénères témoins.

Cependant, la biométrie des organes met en exergue la diminution de $6,60 \%$ du 
poids des reins et l'augmentation de $10,60 \%$ du poids des foies des rats sous régime à $10 \%$ de FAS. Ces modifications de poids d'organes sont statistiquement significatives. Ces organes pourraient donc souffrir d'une pathologie ou d'une perturbation de leur métabolisme nutritionnel. Il est à envisager alors, d'autres moyens de leur exploration pour plus de précision.

\section{REFERENCES}

Abuelo IG. 1990. Renal failure caused by chemicals, foods, plants, animal venom, and misuse of drugs. Arch. Intern. Med., 150: 505 .

Adrian J, Rabache M, Fragne R. 1991. Techniques d'analyse nutritionnelle. In Principes de Techniques d'Analyse. Lavoisier TEC \& DOC (éd) : Paris ; 451478.

Anonyme. 1985. Lowering blood cholesterol to prevent heart disease. Rev. Nutr., 43: 283-285.

Balasubramaiam J. 2003. Neonatal Renal Failure, $3^{\text {rd }}$ Congress of Nephrology in Internet.

Bouafou KGM. 2007. Etude de la production d'asticots à partir d'ordures ménagères et de la valeur nutritionnelle de la farine d'asticots séchés (FAS) chez le rat en croissance. Thèse Doct. Physiol. Anim., Université de Cocody, Abidjan, Côte d'Ivoire, p. 145.

Bouafou KGM, Kouame KG, Offoumou AM. 2007. Bilan azoté chez le rat en croissance de la farine d'asticots séchés. Tropicultura, 25: 70-74.

Bouafou KGM, Zannou-Tchoko V, Konan BA, Kouame KG. 2008. Etude de la valeur nutritionnelle de la farine d'asticots séchés chez le rat en croissance. Rev. Ivoir. Sci. Technol., 12: 215-225.

Brunette MG. 1997. Régulation du métabolisme calcique. In Désordres Acido-basiques et Hydroélectrolytiques,
Offenstadt G, Brunette MG (eds). Arnette Blackwell: Paris ; 294-312.

Budesinky B. 1969. Analytical Chemistry. Chelñates, 18: 5067-5073.

Dingeon B, Ferry JP, Roulet A. 1975. Automatic assay of blood sugar by Trinder's method. Ann. Biol. Clin., 33: 313.

Fawcett JK, Scoot JE. 1960. A rapid and precise method for determination of urea. J. Clin. Path., 13: 156-159.

Finke MD, Defoliart GR, Benevenga NJ. 1989. Use of a four parameter logistic model to evaluate the quality of the protein from three insect species when fed to rats. J. Nutr., 119: 684-691.

Mogensen CE, Keane WF, Bennett PH. 1995. Prevention of diabetic renal disease with special reference to microalbuminuria. Lancet, 346: 1080-1084.

Pawlak M, Pion R. 1968. Influence de la supplémentation des protéines de blé par les doses croissantes de lysine sur la teneur en acides aminés libres du sang et du muscle du rat en croissance. Ann. Biol. Bioch. Biophys, 7: 517-530.

Satish J, Soni GL, Rattan S. 1984. Biochemical and histological studies in albino rats fed on soybean lectin. Nutr. Rep. Intern., 29: 95-106.

Séronie S, Vivien M, Galteau M, Carlier MC, Ahadj A. 2004. Dosage de la créatininémie en 2003 : état des lieux analytique et essai de standardisation de l'étalonnage. Ann. Biol. Clin., 62: 165175.

Williams SR, 1994. Essentials of Nutrition and Diet Therapy ( $6^{\text {th }}$ edn). Masson: New York.

Young DS, Pestaner L, Gibberman V. 1975. Effects of drugs on clinical laboratory tests. Clin. Chem., 21: 5. 